\title{
In Vitro Transmission Electron Microscopy of Water-Borne Dendrimer- Encapsulated Gold Nanoparticles
}

\author{
K.L. Klein, ${ }^{*}$ M.G. Weir, ${ }^{* *}$ R.M. Crooks, ${ }^{* *}$ and I.M. Anderson* \\ *Surface and Microanalysis Science Division, National Institute of Standards and Technology, \\ Gaithersburg MD 20899-8371 \\ **Department of Chemistry and Biochemistry, The University of Texas, Austin TX 78712-0165
}

Due to the growing applications, especially in health-related fields, of functional nanoparticles comprised of both the hard and soft material components, there is rising demand for characterization techniques with the high spatial resolution necessary to image individual such nanostructures in their relevant aqueous ("in vitro") environment. This research addresses the above challenge using a prototype liquid flow cell specimen holder for the transmission electron microscope (TEM) [1]. The high signal and consequent rapid acquisition of TEM bright-field imaging, coupled with the capacity to flow liquid through the probed region, also enables the performance of dynamic experiments, which are currently inaccessible by alternative techniques such as cryo-TEM, and thus allow a unique in situ approach to study particle behavior in a prescribed chemical environment.

Here we demonstrate the utility of this in vitro TEM imaging technique for imaging functional gold (Au) dendrimer-encapsulated nanoparticles (DENs). Bright-field images were acquired with incident energy $E_{0}=300 \mathrm{keV}$, collection angle $\beta=5.4 \mathrm{mrad}$ and energy-selecting slit of width $\Delta E=10 \mathrm{eV}$, using a microfluidic cell comprised of two silicon microchips with electron transparent silicon nitride $\left(\mathrm{SiN}_{\mathrm{x}}\right)$ windows. The microfluidic cell interfaces with a holder designed to provide fluid circulation through the flow cell from outside of the electron microscope, as shown in Fig. 1. Flow through the probed region is demonstrated by the transport of a DEN within the cell relative to stationary 10-nm-diameter Au markers (NIST Reference Material 8011, citrate stabilized) deposited on the outside of the flow cell, as shown in Fig. 2. The imaged DEN is outside of the depth of focus $(\approx 70 \mathrm{~nm})$ centered on the Au markers. We also validate the technique by direct observation of the structure of DENs in their native hydrated state, as shown in Fig. 3. The DENs were synthesized using a two-step process whereby metal ion complexes bound to amine functional groups on the dendrimer are reduced to yield a nanoparticle encapsulated within the dendrimer [2]. Sixth generation hydroxyl-terminated poly(amido amine) (G6-OH PAMAM) dendrimers result in Au nanoparticles with narrow polydispersity and a nominal composition of 147 atoms. The in vitro TEM measurements of the DENs clearly show a dark core with a diameter of $\approx 1.7 \mathrm{~nm}$ within a darkly imaging shell of diameter of $\approx 6.8 \mathrm{~nm}$, in agreement with bulk measurements of each component. The ability to observe directly the attributes of individual nanoparticles and their behavior within their native environments will provide both validation of existing models base on average measurements and otherwise unobtainable information for manifold applications [3].

\section{References}

[1] K.L. Klein, I.M. Anderson, and N. de Jonge, J. Microsc. (2011) available online Jan. 20, 2011. doi:10.1111/j.1365-2818.2010.03484.x

[2] M.G. Weir et al., Langmuir 26 (2010) 1137.

[3] We are grateful to Protochips Inc. for access to their prototype TEM holder and for support from the National Research Council Postdoctoral Research Associateship Program (KLK). 

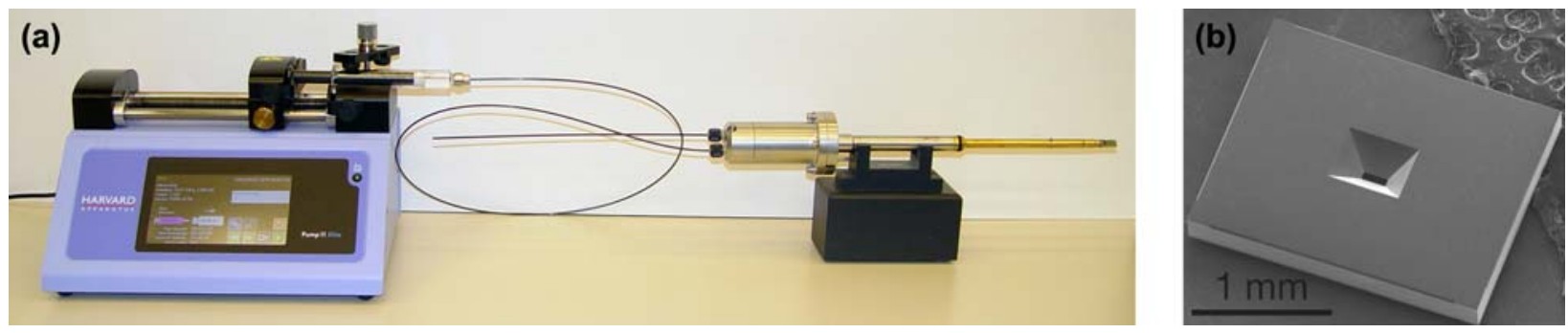

FIG. 1. The liquid flow cell apparatus: (a) photograph of macroscopic components, comprising a syringe pump, syringe, tubing, and TEM holder; (b) scanning electron micrograph of microfluidic chips equipped with electron-transparent $\mathrm{SiN}_{\mathrm{x}}$ windows, which are accommodated by the TEM holder.

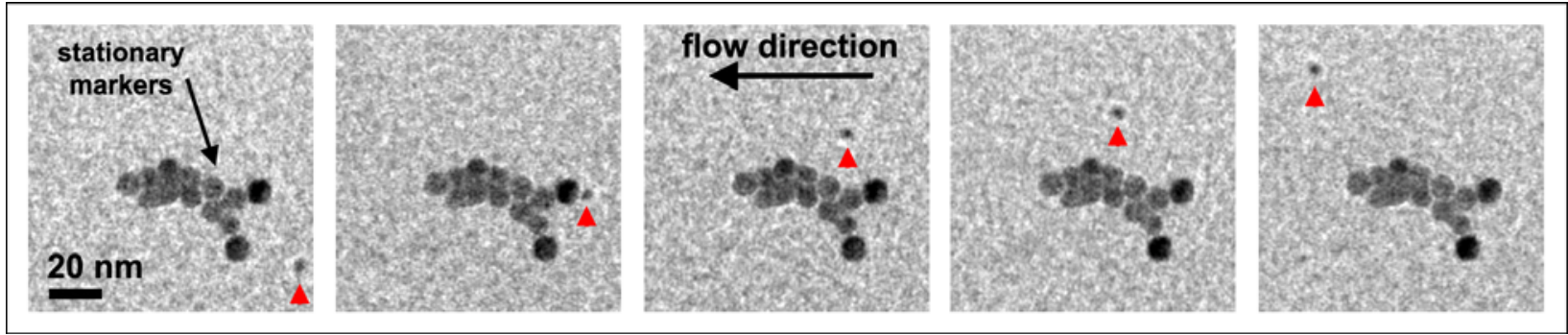

FIG. 2. TEM bright field image sequence showing the transport of a nanoparticle along the flow direction relative to stationary 10-nm-diameter Au nanoparticles, which are deposited on the exterior surface of the $\mathrm{SiN}_{\mathrm{x}}$ windows.

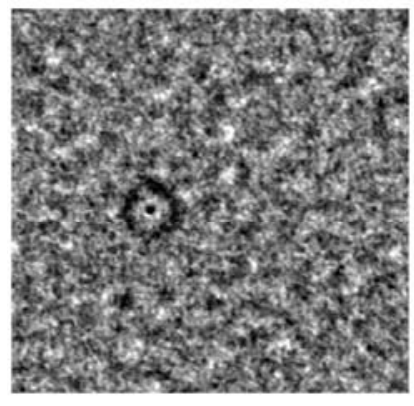

(a) single exposure

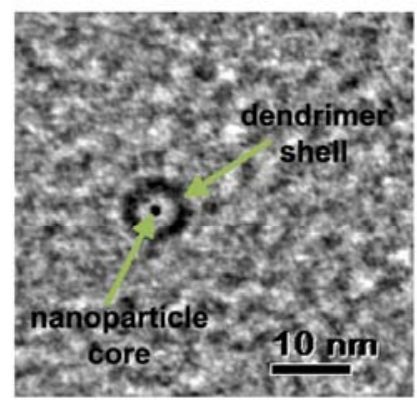

(b) 32 frames summed

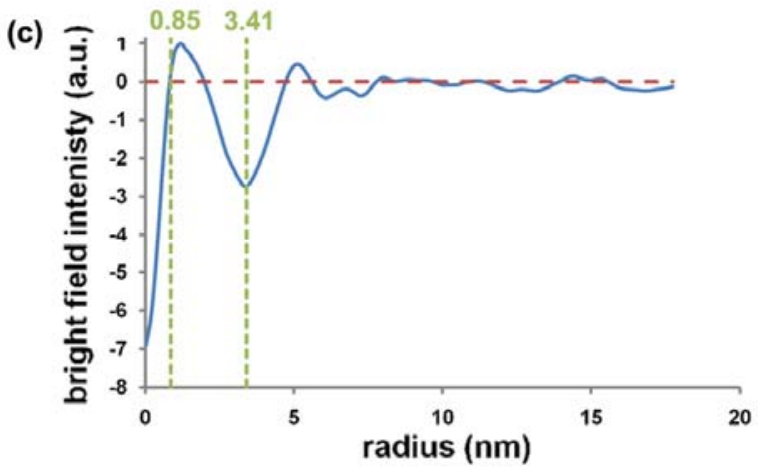

FIG 3. TEM bright field images of a dendrimer-encapsulated nanoparticle (DEN) suspended in $<100 \mathrm{~nm}$ of water: (a) individual $1 \mathrm{~s}$ exposure; (b) sum of 32 sequential $1 \mathrm{~s}$ exposures following manual drift correction to the intensity minimum at the core of the DEN; and (c) plot of radially averaged intensity from (b) relative to the core of the DEN (zero radius) and the mean background intensity (zero relative intensity - see dashed red line). The measured diameters of the core $(1.7 \mathrm{~nm})$ and darkly imaging shell $(6.8 \mathrm{~nm})$ are consistent with the nominal diameters of the 147-atom Au nanoparticles $(1.6 \mathrm{~nm})$ and G6-OH PAMAM dendrimer $(6.7 \mathrm{~nm})$, respectively. 